\title{
Approaches to Disease Modification for Parkinson's Disease: Clinical Trials and Lessons Learned
}

\author{
Albert Y. Hung $^{1}$ (D) Michael A. Schwarzschild ${ }^{1,2}$ \\ Accepted: 30 October 2020 / Published online: 17 November 2020 \\ (C) The American Society for Experimental NeuroTherapeutics, Inc. 2020
}

\begin{abstract}
Despite many clinical trials over the last three decades, the goal of demonstrating that a treatment slows the progression of Parkinson's disease (PD) remains elusive. Research advances have shed new insight into cellular pathways contributing to PD pathogenesis and offer increasingly compelling therapeutic targets. Here we review recent and ongoing clinical trials employing novel strategies toward disease modification, including those targeting alpha-synuclein and those repurposing drugs approved for other indications. Active and passive immunotherapy approaches are being studied with the goal to modify the spread of alphasynuclein pathology in the brain. Classes of currently available drugs that have been proposed to have potential diseasemodifying effects for PD include calcium channel blockers, antioxidants, anti-inflammatory agents, iron-chelating agents, glucagon-like peptide 1 agonists, and cAbl tyrosine kinase inhibitors. The mechanistic diversity of these treatments offers hope, but to date, results from these trials have been disappointing. Nevertheless, they provide useful lessons in guiding future therapeutic development.
\end{abstract}

Key Words Alpha-synuclein · clinical trial $\cdot$ immunotherapy $\cdot$ neuroprotection $\cdot$ repurposing

\section{Introduction}

Parkinson's disease (PD) is a progressive multisystem neurodegenerative disorder that affects both dopaminergic and nondopaminergic systems [1-3]. Neuronal degeneration affecting multiple brain regions leads to the slow but inexorable development of motor and nonmotor symptoms. Based on estimates of PD incidence, the number of cases worldwide is expected to double by 2040 [4, 5]. With the prediction of a "Parkinson pandemic," there is an urgency to find treatments that can reduce the burden of disease.

The greatest unmet need in PD therapeutics is the identification of treatments that can slow the progression of the disease process. Considerable advances have been made in understanding the pathobiology of PD. Both genetic and environmental factors contribute to the development of $\mathrm{PD}$, converging to

Albert Y. Hung

ahung@mgh.harvard.edu

1 Department of Neurology, Massachusetts General Hospital, 55 Fruit Street, Boston, MA 02114, USA

2 MassGeneral Institute for Neurodegenerative Disease, 114 16th Street, Charlestown, MA 02129, USA cause mitochondrial and lysosomal dysfunction, oxidative stress, abnormal protein aggregation, and neuroinflammation $[1,3,6,7]$. Despite these research advances, numerous clinical trials well supported by preclinical and epidemiological data have not demonstrated disease modification. These results have raised important questions about the challenges in identifying potential targets and in study design. Obstacles include the clinical heterogeneity of PD, limitations of preclinical models, and absence of biomarkers of disease progression [8]. Also, extensive neuronal cell loss and reduction in nigrostriatal dopamine precedes the development of signs and symptoms that allow for a diagnosis of PD based on current clinical criteria [9, 10]. Thus, it may be necessary to identify patients with prodromal PD for research trials targeting disease modification [11, 12].

Well-described in a recent review by Lang and Espay [8], the distinction between "neuroprotection" and "disease modification" is also important in considering development of new treatments. While slowing the neurodegenerative process by preventing neuronal cell death is the goal of a putative neuroprotective therapy, this is inherently challenging in the absence of validated research methods to monitor active neuronal loss. As such, developing treatments that modify disease progression regardless of the mechanism may be a more tractable target. 
Despite these challenges, there are numerous treatments in clinical development with the goal to modify PD progression. Based on a recent review of 145 active clinical trials of PD drug therapeutics, 57 (39\%) were considered to be trials of disease-modifying candidates, of which 24 (42\%) were phase 1 studies, $30(53 \%)$ phase 2, and only $3(5 \%)$ phase 3 [13]. Currently, a number of strategies are being actively pursued to identify potential disease-modifying treatments. These approaches can be broadly grouped into those that include targeting $\alpha$-synuclein pathology, repurposing of previously approved drugs, and modifying disease pathways induced by genes associated with familial causes of PD. (These categories are not mutually exclusive, as some treatments target multiple development strategies.) In this review, we discuss the first two areas of focus; therapeutics directed against genetic subtypes of parkinsonism are reviewed elsewhere in this volume [14]. Current clinical trials for disease modification, including those discussed in this review, are presented in Table 1.

\section{Targeting a-Synuclein Pathology}

Progressive accumulation of $\alpha$-synuclein is now accepted to play a principal role in PD pathogenesis. Mutations in the gene encoding $\alpha$-synuclein cause familial forms of PD [24], and abnormal fibrillar aggregates of the protein comprise the core of the Lewy body, the pathologic hallmark of PD [25]. In its normal state, $\alpha$-synuclein is a highly soluble protein enriched at presynaptic terminals that is thought to regulate the release of synaptic vesicles [26]. However, in disease states, it forms toxic oligomers and/or aggregates that localize to the cell body and neurites and contribute to neurodegeneration. The proposed mechanisms by which $\alpha$-synuclein causes toxicity are numerous and beyond the scope of this review, but include dysfunction of organelles including synaptic vesicles, mitochondria, ER and Golgi, lysosomes, autophagosomes, and nuclei; disruption of interorganelle contacts; and dysregulation of axonal transport $[27,28]$. These features of $\alpha$ synuclein pathobiology present targets for various potential therapeutic strategies, including reducing its synthesis, increasing its clearance, or promoting its disaggregation [29]. Use of small interfering RNA to decrease $\alpha$-synuclein expression has been explored in preclinical models [30, 31] but has not advanced to human trials. PBT434 is a novel brain-penetrant small-molecule inhibitor of $\alpha$-synuclein aggregation. In preclinical models, it prevented loss of nigral dopaminergic neurons, reduced $\alpha$-synuclein accumulation, and rescued motor performance [32]. The drug was well tolerated in a phase 1 study [33] and has potential for treating synucleinopathies including PD. Other small molecules targeting $\alpha$-synuclein aggregation or toxicity are currently in early/phase 1 clinical testing. Activation of the $\mathrm{c}-\mathrm{Abl}$ tyrosine kinase pathway has also been proposed to contribute to $\alpha$-synuclein aggregation, and inhibitors are being studied to assess disease-modifying potential (discussed below).

In addition to cell-autonomous mechanisms of toxicity, there is a growing body of experimental evidence to suggest that $\alpha$-synuclein can spread by cell-to-cell transmission [34]. Numerous studies using cellular and animal PD models have demonstrated that $\alpha$-synuclein aggregates can pathologically spread to adjacent neurons, and that synthetic $\alpha$-synuclein fibrils or brain extracts containing Lewy bodies can induce formation of new inclusions and loss of dopaminergic neurons [35-38]. This model is also supported by the discovery of host-to-graft spread of Lewy body pathology into fetal cells transplanted into the brains of humans with PD [39, 40]. The ability of $\alpha$-synuclein to form aggregates by templated misfolding and disseminate between cells has led to the concept that a prion-like mechanism may contribute to PD [34, 38].

Active and passive immunotherapies are currently being pursued as therapeutic strategies to target extracellular $\alpha$ synuclein and impede cell-to-cell transmission [41]. Results from a first in-human, randomized phase 1 study of a novel therapeutic (PD01A) involving immunization with a short peptide mimicking a $\mathrm{C}$-terminal epitope of human $\alpha$ synuclein were recently reported, combining four sequential studies [15]. Subjects were initially randomized to receive four subcutaneous immunizations at either low dose $(15 \mu \mathrm{g})$ or high dose $(75 \mu \mathrm{g})$ every 4 weeks, followed for a period of 25-35 months, re-randomized to either low-dose or high-dose booster immunization, and then re-immunized with a second booster injection (high dose) when the antibody response was close to baseline; 21 out of 24 subjects completed all treatments. Treatment was considered safe and well tolerated, with the majority of adverse events deemed unrelated to the study agent (apart from local injection site reactions). Active immunization induced a specific immune response as determined by serum antibody concentration. There was no change in CSF total $\alpha$-synuclein levels, although post hoc analysis revealed a $51 \%$ decrease in CSF oligomeric species in those who received high-dose treatment. Although this study did not include a placebo control, it provides safety and pharmacodynamic support for conducting additional active immunotherapy trials.

Passive immunotherapy using antibodies that target specific epitopes of $\alpha$-synuclein is currently an exciting area of therapeutic development. Two therapies have now progressed to phase 2 trials. PRX002/RG7935 (prasinezumab), developed by Prothena and Roche, is a humanized monoclonal antibody that targets the $\mathrm{C}$-terminus of $\alpha$-synuclein. In PD mouse models, this antibody promotes $\alpha$-synuclein clearance and reduces behavioral deficits in motor tasks [42]. In a randomized, double-blind, placebo-controlled multiple ascending dose trial, prasinezumab was safe and well-tolerated with dose-dependent increases in CSF levels, though they were 


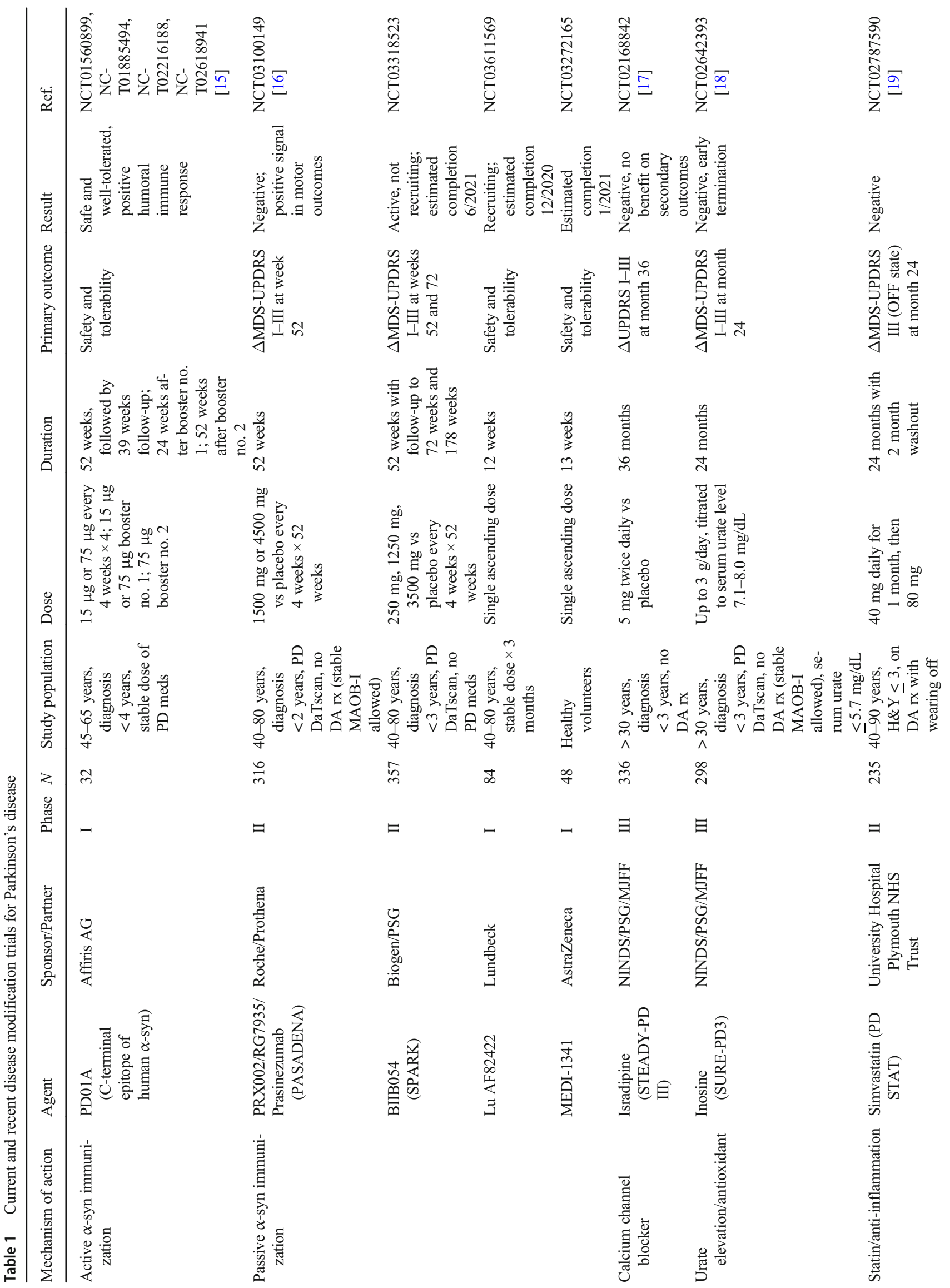




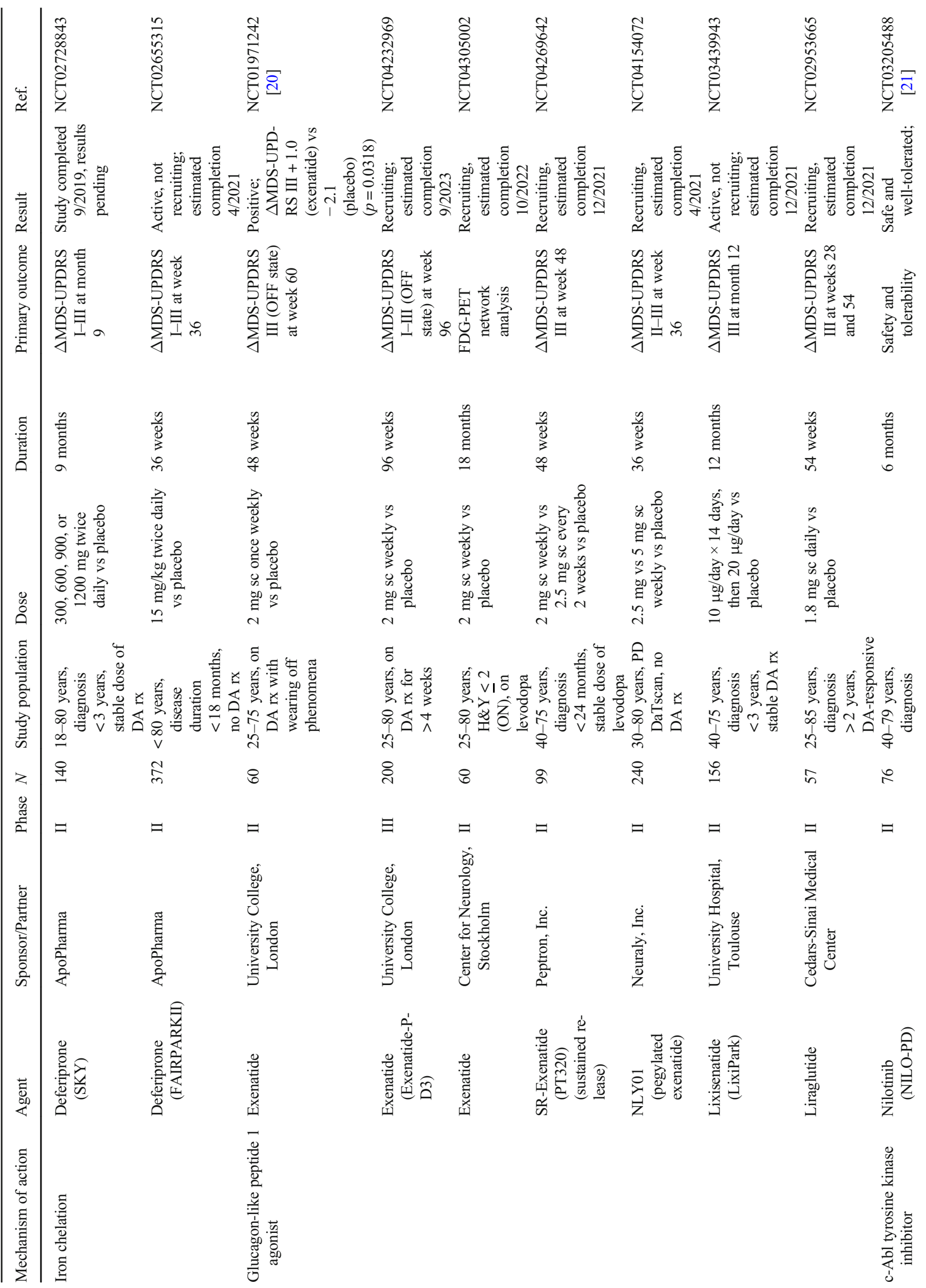




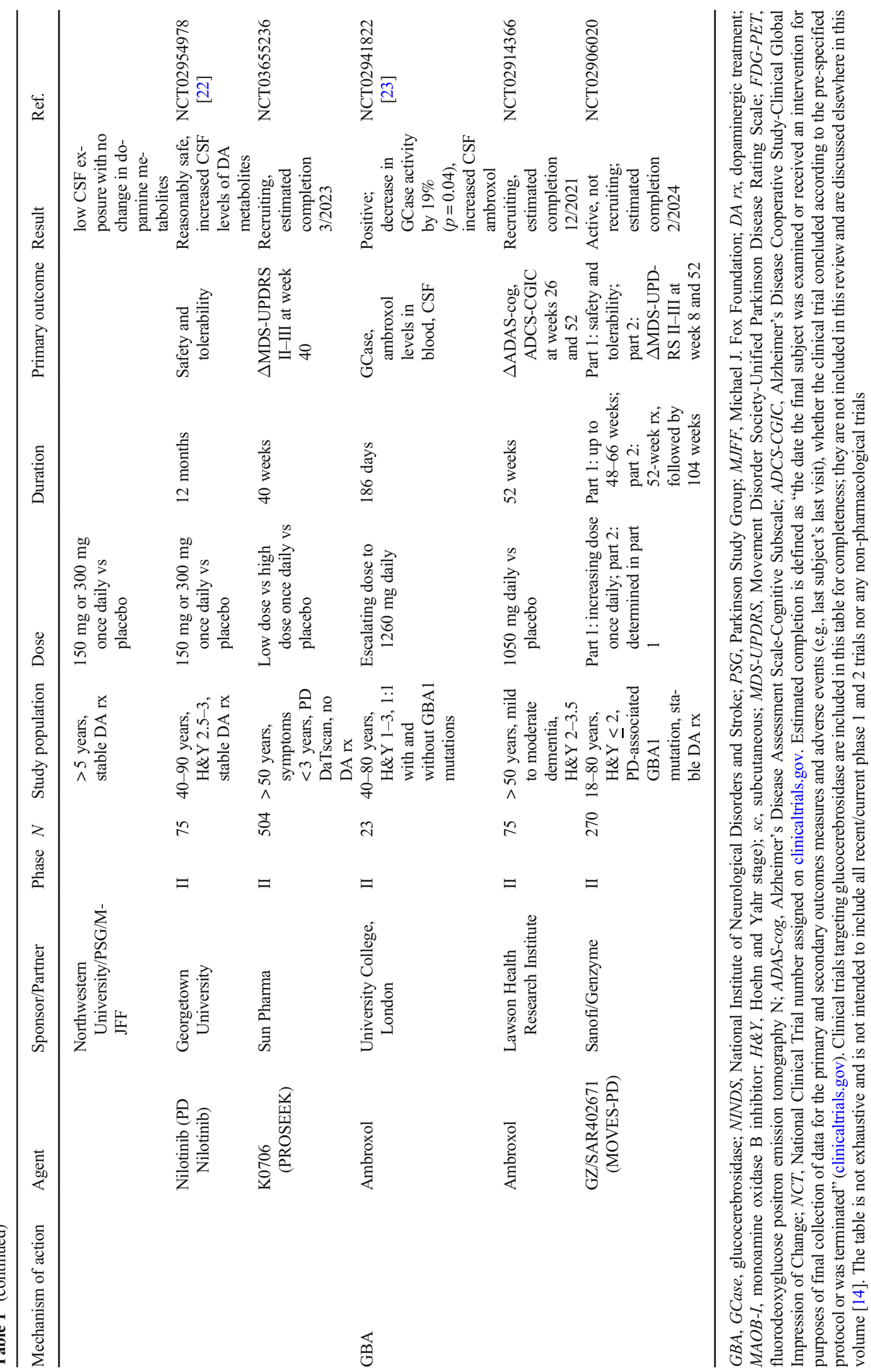


only $\sim 0.3 \%$ of those achieved in serum [43]. These studies led to the design of the PASADENA trial, a phase 2 randomized trial of 316 subjects with early $\mathrm{PD}$, in which doses of up to $4500 \mathrm{mg}$ were administered every 4 weeks over 52 weeks. While results from this trial have not yet been published, recently presented data indicate that the study did not meet its primary outcome measure of a change from baseline in the MDS-UPDRS total score (parts I-III) at week 52 [16]. However, signals of efficacy were detected in the rate of decline in motor function and time to worsening of motor symptoms. A blinded extension phase, PASADENA Part 2, is currently ongoing.

A second monoclonal antibody, BIIB054, is undergoing clinical development by Biogen and is also in a phase 2 trial. This antibody targets an $\mathrm{N}$-terminal epitope of $\alpha$-synuclein, preferentially recognizes aggregated species of the protein, and prevents spread in preclinical models [44]. In a phase 1 study of healthy volunteers and participants with PD, treatment was safe and well-tolerated over a period of 16 weeks [45]. A phase 2 trial (SPARK) recently achieved primary completion with an estimated study completion date of June 2021.

Several additional passive immunotherapy treatments are currently being studied in phase 1 trials, including LuAF82422 (Lundbeck) and MEDI-1341 (AstraZeneca). These are listed in the clinicaltrials.gov database, but limited peerreviewed data are available. A phase 1 safety and tolerability study of the monoclonal antibody ABBV-0805 (Abbvie) was withdrawn as of June 2020.

\section{Repurposing Drugs for Parkinson's Disease}

In light of the current understanding of PD pathogenesis, identifying treatments that directly target $\alpha$-synuclein is a worthy and critical goal. However, new drug discovery and development is a lengthy, very expensive process with potential risk of failure. A recent investigation estimated the median research and development cost of bringing a drug to market at approximately $\$ 1$ billion and the total mean investment at $\$ 1.3$ billion [46]. In addition, it can take 10 years or more to develop a drug from early-phase preclinical studies through phase 3 trials. Disease modification trials for neurodegeneration are particularly challenging because they often require patients to be followed for multiple years.

Because of high attrition rates, increased cost, and the slow pace of discovery associated with development of novel therapeutics, drug repurposing (also called drug repositioning) is a viable strategy to identify possible new treatments. In this approach, existing drugs are applied to new therapeutic uses outside its original medical indication [47]. Potential advantages of drug repurposing over developing an entirely new drug include the following: lower risk of failure due to safety issues; reduced time frame for development as most of the preclinical testing and safety assessments have already been completed; and less investment in preclinical and early-phase costs [48]. Supported by preclinical, epidemiological, and clinical evidence, numerous drugs have been repurposed as putative disease-modifying agents for PD. In this section, we review some of the classes of medication that either are being studied actively in clinical trials or have recently completed investigation.

\section{Calcium Channel Blockers}

Neurodegeneration in PD is determined not only by genetic and environmental influences, but also by selective vulnerability of a subset of neuronal populations, including nigral dopaminergic neurons. The cell-autonomous mechanisms accounting for these differences are not well understood. However, neurons that rely on $\mathrm{Ca}(\mathrm{v}) 1.3 \mathrm{~L}$-type calcium channels to maintain autonomous pacemaking function may be particularly susceptible to mitochondrial oxidative stress, raising the possibility that inhibition of these channels may be protective [49]. Isradipine, a dihydropyridine calcium channel blocker with the highest affinity for $\mathrm{Ca}(\mathrm{v}) 1.3$ channels and approved for treatment of hypertension, indeed demonstrated neuroprotective effects in animal models of PD [50, 51]. In addition, epidemiologic studies have shown that use of calcium channel blockers of all types is associated with an overall significantly decreased risk of PD [52].

Based on these preclinical findings, a phase 2 randomized clinical trial was conducted to establish dose tolerability [53]. This trial identified a dose of $10 \mathrm{mg}$ daily as the maximal tolerable dose, with higher doses associated with adverse effects including peripheral edema and dizziness. Using this dose, a large phase 3 multicenter, randomized, parallel-group, double-blind, placebo-controlled trial to assess the effect of isradipine on clinical progression (STEADY-PD III) was conducted to assess efficacy [17]. In this study, 336 patients with early PD not yet taking dopaminergic medications were randomized to isradipine or placebo for a period of 36 months. The primary outcome was change in the UPDRS parts I to III score measured in the "ON" state between baseline and 36 months. Treatment with isradipine failed to confer any benefits on clinical progression, with no difference in both primary and secondary outcome measures [17]. Whether more potent inhibition of calcium channels or intervention at earlier prodromal stages of $\mathrm{PD}$ would alter disease progression remains unanswered.

\section{Antioxidant and Anti-inflammatory Agents}

Mitochondrial dysfunction and oxidative stress have long been postulated to play a role in PD pathogenesis [54, 55]. The brain is uniquely susceptible to oxidative injury given its high level of energy consumption with resulting generation of 
reactive oxygen species. Moreover, dopamine metabolism by monoamine oxidase itself contributes to the generation of toxic free radicals. Postmortem studies of PD brains demonstrate evidence of oxidative stress, including deficiency of mitochondrial complex I activity, decreased levels of antioxidant enzymes and glutathione, and higher levels of lipid peroxidation products. Many genes associated with familial PD also encode proteins that are directly involved in mitochondrial function, including $\alpha$-synuclein, parkin, PTEN-induced kinase 1 (PINK1), DJ-1, leucine-rich repeat kinase 1 (LRRK2), and vacuolar protein sorting-associated protein 35 (VPS35) [55, 56].

Despite the preponderance of scientific evidence implicating these mechanisms in PD, clinical trials targeting oxidative stress to date have been disappointing. Completed more than 25 years ago, the Deprenyl and Tocopherol Antioxidative Therapy of Parkinsonism study (DATATOP), the first disease modification trial in PD, failed to show benefit for vitamin E [57]. More recently, a large randomized trial of high doses of coenzyme Q10 in combination with vitamin E also did not show any effect on clinical progression [58]. Peroxisome proliferator-activated receptor gamma (PPAR $\gamma$ ) is a ligandgated transcription factor that regulates redox balance, mitochondrial function, and inflammatory response. The PPAR $\gamma$ agonist pioglitazone, available as a treatment for diabetes, reduces neuroinflammation and neurodegeneration in animal models [59]. However, in a 44-week randomized trial, pioglitazone at doses of $15 \mathrm{mg} /$ day and $45 \mathrm{mg}$ /day was found to be futile in modifying disease progression [60].

Although pathological studies implicate oxidative stress mechanisms in PD, case-control studies have not demonstrated a difference between PD patients and age-matched controls for the majority of endogenous antioxidant molecules or enzymes. One exception is urate, the enzymatic end product of purine metabolism which accounts for $60 \%$ of total plasma antioxidant activity [61]. Multiple prospective epidemiological studies have shown that patients with higher plasma urate concentration have a lower risk of developing PD, particularly in men [62, 63]. In addition, higher baseline urate levels were associated with significantly slower rates of disease progression, based on data from two large clinical studies of early PD $[64,65]$. Urate also demonstrated neuroprotective effects in cellular and animal PD models [61].

Inosine, a nucleoside and purine precursor of urate and available as a dietary supplement, has been studied for its potential to elevate urate levels and modify PD progression. In a phase 2 study, inosine titrated to elevate serum urate concentration to a mildly $(6.1-7.0 \mathrm{mg} / \mathrm{dl})$ or moderately elevated $(7.1-$ $8.0 \mathrm{mg} / \mathrm{dl}$ ) level for up to 24 months was clinically safe and well-tolerated [66]. Treatment also effectively raised CSF levels of urate. A phase 3 randomized clinical trial was conducted to determine whether oral inosine dosed to moderately elevate serum urate (from $<5.7 \mathrm{mg} / \mathrm{dl}$ to $7.1-8.0 \mathrm{mg} / \mathrm{dl}$ ) over
2 years slows clinical decline in early PD (SURE-PD3). The primary outcome was rate of change in the MDS-UPDRS I-III total score at 24 months compared to baseline. However, the study was stopped prior to its planned completion date as interim analysis indicated that it would be unable to show efficacy based on its primary outcome measure and timeline [18].

Trials continue to examine the potential use of antioxidants to slow disease progression. $\mathrm{N}$-acetylcysteine (NAC) is a natural supplement that raises levels of the intracellular antioxidant glutathione and is used as a mucolytic agent for patients with pulmonary disease and as a treatment for acetaminophen overdose. In a recent small single-center open-label study, patients randomized to receive weekly infusions $(50 \mathrm{mg} / \mathrm{kg})$ and oral doses ( $500 \mathrm{mg}$ twice a day) of NAC for 3 months had increased dopamine transporter (DAT) binding (mean increase from 3.4 to $8.3 \%$ ) compared with controls as well as improved PD symptoms [67]. Larger-scale studies are warranted to explore these results.

Statins have also been explored as potential disease modifiers of PD. Inhibitors of 3-hydroxy-3-methylglutaryl coenzyme A (HMG-coA) reductase, statins are widely prescribed as cholesterol-lowering agents. They also have multiple biochemical actions independent of cholesterol, including anti-inflammatory effects [68]. In PD models, they have been proposed to exert neuroprotective effects through an anti-inflammatory response, improving motor function and attenuating increase in inflammatory cytokines $[69,70]$. Simvastatin is effective in crossing the blood-brain barrier and is currently being studied in a phase 2 randomized, placebo-controlled futility trial. Unfortunately, recently announced results indicated futility for slowing progression of PD, and thus do not warrant further development of simvastatin for this indication [19].

\section{Iron-Chelating Agents}

Excessive iron deposition in the substantia nigra of patients with PD has been demonstrated by postmortem analysis and iron-sensitive high-field MRI imaging [71]. Iron leads to increased production of reactive oxygen species and may induce ferroptosis, an iron-dependent mechanism independent of other cell death pathways [72]. Deferiprone is an iron-chelating agent approved for the treatment of iron overload in hematologic conditions such as thalassemia major and is able to penetrate the blood-brain barrier. In cell culture and MPTP mouse models of PD, treatment with deferiprone reduced oxidative stress and improved motor symptoms while raising striatal dopamine [73]. A small pilot study using a randomized delayed-start paradigm in patients with early PD suggested that early treatment with deferiprone reduced iron deposits in the substantia nigra and improved motor function [73]. In another randomized, double-blind, placebo-controlled study, 
treatment with deferiprone for 6 months was well tolerated and reduced iron content in specific brain regions [74].

These findings have prompted additional larger efficacy trials. One dose-ranging study randomized 140 patients with early PD on a stable dose of dopaminergic therapy to placebo or a range of deferiprone doses from 600 to $2400 \mathrm{mg}$ daily (SKY). The phase 2 trial used the change in the motor subscale (part III) of the MDS-UPDRS as the primary outcome measure. The study was completed as of September 2019 and results are pending. A second phase 2 trial of 372 early untreated PD patients is ongoing, comparing treatment with deferiprone $30 \mathrm{mg} / \mathrm{kg} /$ day with placebo (FAIR PARK II). Using a primary outcome of change in total MDS-UPDRS between baseline and 36 weeks, this trial is active but not recruiting with an estimated study completion date of April 2021. The results from these studies will be helpful in guiding future investigations of iron chelation as a therapeutic strategy for PD.

\section{Glucagon-Like Peptide 1 Agonists}

There is increasing interest in a potential role for glucagonlike peptide 1 (GLP-1) agonists in the treatment of neurodegenerative diseases including PD. GLP-1 acts to regulate glucose homeostasis and facilitate insulin signaling. As such, compounds that activate the GLP-1 receptor, including GLP-1 analogs and inhibitors of dipeptidyl peptidase 4 (DPP-IV), are used in the treatment of type 2 diabetes mellitus. In the brain, GLP-1 signaling has been proposed to influence myriad processes that affect neuronal survival [75]. Insulin resistance has also been implicated as a contributor to neurodegeneration [76]. A large retrospective cohort study reported an increased risk of subsequent PD following type 2 diabetes [77], although other studies have shown positive, no, or negative associations [78-82] and no study has linked GLP-1 or DPP-IV levels or activity to PD risk or progression.

Endogenous GLP-1 is rapidly degraded by DPP-IV into inactive metabolites. However, a naturally occurring GLP-1 agonist that is resistant to DPP-IV cleavage, exendin-4, has been used in preclinical and clinical studies [83]. In animal models of PD, exenatide, a synthetic form of exendin-4, prevented microglial-mediated conversion of astrocytes into an A1 neurotoxic phenotype, protecting against loss of dopaminergic neurons and reducing behavioral deficits [84]. Other neuroprotective mechanisms have also been proposed, including beneficial effects on mitochondrial function, synaptic plasticity, neurogenesis, and enhancing the actions of brain-derived neurotrophic factor [75].

The clinical effects of exenatide on PD have been studied thus far in two clinical trials. In an initial single-blind proof-ofconcept trial, 44 patients with moderate-stage PD were treated with either subcutaneous exenatide (10 mcg twice daily) or placebo for 12 months. Apparent benefits were reported to persist in motor and cognitive function at 14 months, despite patients being off exenatide during a 2-month washout period [85]. A subsequent double-blind, placebo-controlled trial of 60 patients with earlier stage disease (mean duration 6.4 years) was conducted, using $2 \mathrm{mg}$ subcutaneous exenatide once weekly for 48 weeks as the active treatment arm. The primary outcome measure, change in MDS-UPDRS motor subscale (part 3) score at 60 weeks (following 12 weeks off treatment), demonstrated modest but statistically significant improvement in motor symptoms in the treated group, though scores were actually worse in the exenatide group at 60 weeks due at least in part to baseline differences despite randomization [20]. Treatment was well-tolerated except for mild weight gain. It is noteworthy that while exenatide is proposed to have a putative neuroprotective effect, the apparent early benefits noted in this trial, which were fully manifest by 12 weeks of treatment, raise the possibility of the drug having symptomatic benefit, further complicating interpretation of the results. Despite their limitations, these studies are encouraging and warrant further investigation that addresses questions about study design, stage of disease, and dosing.

Multiple randomized, double-blind, placebo-controlled studies of GLP-1 agonists are currently ongoing, including a multicenter trial of a novel pegylated form of exenatide (NLY01) in untreated patients with early PD, a multicenter study of sustained-release exenatide in early PD patients (PT320), and several single-center trials of patients on dopaminergic therapy. Other GLP-1 agonists that are under active investigation include lixisenatide and liraglutide. Taken together, these studies should provide valuable insights into whether activation of GLP-1 mediated pathways can modify PD progression.

\section{c-Abl Tyrosine Kinase Inhibitors}

$\mathrm{c}-\mathrm{Abl}$ is a member of the Abl family of non-receptor tyrosine kinases with diverse biological functions [86]. In the central nervous system, it plays an important role in regulating synapse formation, neurite outgrowth, and neurogenesis. Notably, c-Abl is activated in an age-dependent manner, and increased levels of activated c-Abl are present in the substantia nigra and striatum of PD postmortem brains and in toxin-based and transgenic mouse models. Notably, aberrant activation of c-Abl also contributes to accumulation of toxic $\alpha$-synuclein species [87].

Nilotinib is a small-molecule inhibitor of c-Abl tyrosine kinase that is approved for the treatment of chronic myelogenous leukemia. In the MPTP model of PD, administration of nilotinib reduced c-Abl activation and prevented loss of dopamine neurons and behavioral deficits [88]. An initial openlabel pilot study of 12 patients with advanced PD showed mild improvements in motor and cognitive function at 24 weeks of 
treatment, although the observed improvements were reversed by 36 weeks [89]. This study generated considerable excitement, but interpretation was limited by the small size, the absence of a control arm, and the confound of monoamine oxidase-B inhibitor withdrawal potentially accounting for the highlighted biomarker change of increased CSF levels of the dopamine metabolite homovanillic acid [90].

Since that time, two additional randomized phase 2 clinical trials have been conducted comparing nilotinib at doses of $150 \mathrm{mg}$ and $300 \mathrm{mg}$ daily with placebo to determine safety and tolerability. A single-center study of 75 patients was reported to show increased levels of dopamine metabolites in the CSF of patients treated with one of four doses of nilotinib. No significant differences were observed in motor and nonmotor outcomes between the groups, although the study was not powered to assess efficacy. Although the total number of adverse events was similar, there was a higher number of serious adverse events in patients treated with nilotinib, including four cardiovascular events [22]. However, a multicenter trial (NILO-PD) with similar study design yielded conflicting results. Although it also did not test efficacy, nilotinib was found to have poor CNS penetration and did not change dopamine metabolites [21].

The conflicting results bring into question whether future trials of nilotinib are to be pursued. However, they do not rule out the possibility that other inhibitors of the c-Abl pathway may be more effective. A phase 2 study is currently recruiting patients with early PD not yet on symptomatic treatment for randomization to two doses of an experimental Abl inhibitor K0706 compared to placebo (PROSEEK). The study will enroll 504 patients and the primary outcome measure will be the change in MDS-UPDRS parts 2 and 3 after 40 weeks of treatment. The estimated study completion date is 2021 .

\section{Discussion}

In this review, we provide an update on recent and current disease modification trials, focusing on those that target $\alpha$ synuclein as well as those that repurpose currently available therapies. The plethora of active clinical studies offers promise in finding treatments beyond currently available symptomatic therapy.

Nevertheless, caveats exist with both of these developmental strategies. PD is associated with complex pathophysiologic changes, many of which predate onset of clinical motor symptoms by years or decades. Abnormal aggregation of $\alpha$ synuclein is thought to play a pivotal role early in the disease process, triggering other processes such as oxidative stress, mitochondrial dysfunction, autophagy, and neuroinflammation. Thus, even at the earliest stages of symptomatic PD, the underlying pathogenic cascade may be too far advanced for $\alpha$-synuclein-directed therapies to meaningfully modify disease progression.

In contrast, some treatments that are repurposed for PD may target mechanisms that are less specific to the disease process. The theoretical advantage of this approach is potential savings in cost and time for drug development. In reality, however, some of these theoretical benefits may be difficult to achieve. For example, the time exigencies and efficiencies characteristic of pharmaceutical industry programs are not easily replicated in non-commercial development, as multiple grant cycles typically add years onto transitions from phase 2 to phase 3 studies when relying on foundation and government funding. For example, 3.5 years passed between the completion date of a successful phase 2 trial of inosine (December 2012) $[66,91]$ and the start date of the resulting phase 3 trial (June 2016) [18] despite uninterrupted investigator-driven efforts for a prompt transition (MAS, unpublished observations). Similarly, a safety profile established at a given dose and duration for an approved therapeutic indication may not be sufficient without additional preclinical and clinical safety data to support a new indication for long-term disease modification in PD.

In considering the current studies reviewed here, it is important to evaluate them in the context of prior studies. The "failure" to identify any disease-modifying therapies to date is sobering. Despite compelling preclinical data and thoughtful study design, long, costly trials have not yielded any clearly positive results. Nevertheless, these studies provide useful lessons in guiding future research. (A detailed discussion of clinical trial design is beyond the scope of this review but has been thoughtfully considered elsewhere [92-94].)

\section{Lessons Learned for Target Selection}

While strong preclinical evidence from cell-based and animal models remains critical to effective therapeutic development, this has thus far not been sufficient to lead to effective treatments. Nevertheless, recent trials with disappointing results provide useful lessons in guiding further development. One step forward is the use of human epidemiological data to provide additional support for target selection. For example, despite the lack of efficacy for isradipine in the STEADY-PD III trial, the lower risk of PD associated with calcium channel blocker use identifies this treatment as a worthy target [17, 52]. Identification of serum urate as a clinical biomarker associated with slower disease progression provides an even stronger example of using epidemiology to motivate target development [64, 65]. Although inosine did not have a disease-modifying effect [18], this approach nonetheless strengthens the motivation to move potential targets through the clinical pipeline.

Trials targeting $\alpha$-synuclein highlight the use of genetics to guide development of new therapeutics. A central role for $\alpha$ - 
synuclein in PD pathogenesis was initially discovered based on identification of gene mutations causing familial PD. Extensive research has since increased understanding of $\alpha$-synuclein trafficking and aggregation, leading to the identification of new druggable targets and immunological approaches. PD genetics are also likely to provide the groundwork for the next phase of disease modification trials [14].

In addition, recent trials have established the importance of including measures to demonstrate that the drug is able to penetrate the central nervous system at pharmacologically meaningful levels and to engage the target of interest. As examples, the demonstration that active immunotherapy against $\alpha$-synuclein may influence CSF oligomeric species [15] and that antibodies used in passive immunotherapy trials are detected in CSF [43] provides important justification that further development may yield positive results. Considerable advances have been made to measure different forms of $\alpha$ synuclein in biofluids and peripheral tissues [95] and to image $\alpha$-synuclein neuropathology in living humans [96]. Indeed, the Michael J. Fox Foundation recently awarded several large grants in pursuit of an $\alpha$-synuclein imaging tracer [97]. When and if these biomarkers are available, they may greatly improve assessment of target engagement and potentially provide a complementary and ultimately surrogate measure of efficacy for disease modification.

Other recent trials have similarly demonstrated efficacy in penetrating the CNS and engaging the putative target. Use of inosine to elevate serum urate results in increased CSF levels [66], and deferiprone was shown to reduce brain iron deposition $[73,74]$. While these findings are not necessarily predictors of disease modification, they may be prerequisites and highlight the progress in study design compared to early trials, which did not include measures of target engagement. The recently completed multicenter nilotinib trial further demonstrates the importance of these measures as the low level of CNS drug penetration may provide a pharmacodynamic explanation for the lack of benefit [21].

\section{Lessons Learned for Trial Design}

Steps to improve clinical trial design, including subject selection and outcome measurement, will also enhance the likelihood of success in future disease modification trials. To improve subject selection, DAT imaging is now being used as an enrichment biomarker for clinical trials targeting early PD [98]. Previous trials in which enrollment was based on clinical diagnosis alone included subjects who had "scans without evidence of dopaminergic deficit" (SWEDD). Subjects in this group, which may comprise up to $10-15 \%$ in some early PD cohorts, may either not have PD or have very slow progression [99-101], and are difficult even for movement disorder specialists to distinguish clinically from those with abnormal
DAT scans [102]. Exclusion of patients without evidence of dopamine deficiency may enrich clinical trial populations for subjects who are likely to progress clinically and improve statistical power. SURE-PD3 was the first randomized clinical trial to include DAT imaging as an eligibility criterion, and this is now included in subject screening in numerous trials.

Given concerns that patients with early symptomatic PD already demonstrate measurable dopaminergic cell loss, there is now considerable interest in identifying patients with prodromal PD [103-105]. Research criteria for prodromal PD have been proposed $[11,12]$, raising prospects of using potential disease-modifying therapies prior to the development of motor symptoms. Alternatively, identification of individuals who carry genetic forms of PD can enrich clinical trials for those with the very earliest stage of disease ([14]).

Progress has also been made in the refinement of rating scales to measure PD symptoms. Change over time in the MDS-UPDRS is now routinely used as the primary outcome measure for PD disease modification trials. Compared to the original UPDRS, this scale allows for differentiation between relatively milder impairments encountered in early stages of PD and more broadly assesses both nonmotor and motor features of the disease, as reported by patients as well as clinicians [106]. Although now widely utilized, the MDS-UPDRS was not accepted as a clinical outcome until recent years. Indeed, STEADY-PD III was the first interventional trial in a de novo population to systematically evaluate the MDSUPDRS [107].

Improvement in detecting progression of motor symptoms may also enhance clinical trials in the future. Digital biomarkers are being explored as measures of motor function that will allow for more frequent or continuous, patient-centered monitoring than the usual study visit-based clinical assessments $[108,109]$. AT-HOME PD is an observational study to assess long-term clinical outcomes in PD patients who participated in the STEADY-PD III and SURE-PD3 clinical trials (NCT03538262). Recruiting patients with early, untreated PD, the WATCH-PD study (NCT03681015) is designed to compare information collected from wearable sensory and mobile devices with traditional clinical assessments and rating scales. These approaches may refine the ability to detect smaller clinical changes early in the disease process.

The clinical heterogeneity of PD has also been postulated to contribute to the lack of demonstrated benefit in disease modification trials. Another lesson learned may be the use of specific subpopulations who are most likely to benefit from a specific therapy. In the SURE-PD3 study, only those subjects with a baseline serum urate below the population mean and most likely to respond were included. A similar approach is now being used in studies targeting genetic forms of PD.

The results from recent trials, while disappointing in the short term, offer promise for the future. The need to identify disease-modifying therapies remains as great as ever, perhaps 
even more critical now given the growing burden of PD. Indeed, these studies, even those that are "negative," should not be viewed as failures but as steps toward this goal. Advances in developing new biomarkers to monitor disease progression and target engagement and identifying prodromal or at-risk populations, guided by lessons learned from current studies, will hopefully pave the way for more successful trials in the future.

Supplementary Information The online version contains supplementary material available at https://doi.org/10.1007/s13311-020-00964-w.

Acknowledgments Work on this review was supported by NIH grants U01NS09025 and RO1NS110879, and the Farmer Family Foundation Parkinson's Research Initiative (M.A.S.).

Required Author Forms Disclosure forms provided by the authors are available with the online version of this article.

\section{References}

1. Kalia LV, Lang AE. Parkinson's disease. Lancet 2015;386(9996): 896-912.

2. Reich SG, Savitt JM. Parkinson's Disease. Med Clin North Am 2019;103:337-350.

3. Simon DK, Tanner CM, Brundin P. Parkinson Disease Epidemiology, Pathology, Genetics, and Pathophysiology. Clin Geriatr Med 2020;36:1-12.

4. Dorsey ER, Bloem BR. The Parkinson Pandemic-A Call to Action. JAMA Neurol 2018;75:9-10.

5. Dorsey ER, Sherer T, Okun MS, Bloem BR. The Emerging Evidence of the Parkinson Pandemic. J Parkinsons Dis 2018;8:S3S8.

6. Senkevich K, Gan-Or Z. Autophagy lysosomal pathway dysfunction in Parkinson's disease; evidence from human genetics. Parkinsonism Relat Disord 2020;73:60-71.

7. Thomas R, Hallett PJ, Isacson O. Experimental studies of mitochondrial and lysosomal function in in vitro and in vivo models relevant to Parkinson's disease genetic risk. Int Rev Neurobiol 2020;154:279-302.

8. Lang AE, Espay AJ. Disease Modification in Parkinson's Disease: Current Approaches, Challenges, and Future Considerations. Mov Disord 2018;33:660-677.

9. Postuma RB, Berg D, Stern M, et al. MDS clinical diagnostic criteria for Parkinson's disease. Mov Disord 2015;30:1591-1601.

10. McCann H, Cartwright H, Halliday GM. Neuropathology of $\alpha$ synuclein propagation and braak hypothesis. Mov Disord 2016;31:152-160.

11. Berg D, Postuma RB, Adler CH, et al. MDS research criteria for prodromal Parkinson's disease. Mov Disord 2015;30:1600-1611.

12. Heinzel S, Berg D, Gasser T, et al. Update of the MDS research criteria for prodromal Parkinson's disease. Mov Disord 2019;34: 1464-1470.

13. McFarthing K, Buff S, Rafaloff G, Dominey T, Wyse RK, Stott SRW. Parkinson's Disease Drug Therapies in the Clinical Trial Pipeline: 2020. J Parkinsons Dis 2020;10:757-774.
14. Schneider SA, Hizli B, Alcalay RN. Emerging targeted therapeutics for genetic subtypes of parkinsonism [published online ahead of print, 2020 Sep 10]. Neurotherapeutics 2020;1-15. https://doi. org/10.1007/s13311-020-00920-8

15. Volc D, Poewe W, Kutzelnigg A, et al. Safety and immunogenicity of the $\alpha$-synuclein active immunotherapeutic PD01A in patients with Parkinson's disease: a randomised, single-blinded, phase 1 trial. Lancet Neurol 2020;19:591-600.

16. Pagano G, Taylor K, Cabrera J, et al. PASADENA: A Phase 2 study to evaluate the safety and efficacy of prasinezumab in early Parkinson's disease; Part 1 Week-52 results. Mov Disord 2020;35 (suppl 1):S410-11. Abstract.

17. Parkinson Study Group STEADY-PD3 Investigators. Isradipine versus placebo in early Parkinson disease: a randomized trial. Ann Intern Med 2020;172:591-598.

18. ClinicalTrials.gov [Internet]. Bethesda (MD): National Library of Medicine (US). 2015 Dec 30 -. Identifier NCT02642393. Study of Urate Elevation in Parkinson's Disease, Phase 3 (SURE-PD3); $2020 \mathrm{Jul} 28$ [cited 2020 Oct 22]. Available from: https:// clinicaltrials.gov/ct2/show/results/NCT02642393

19. The Cure Parkinson's Trust. The PD-STAT/Simvastatin results [Internet]. London: The Cure Parkinson's Trust; 15 Sep 2020 [cited 2020 Oct 22]. Available from: https://www.cureparkinsons. org.uk/news/the-pd-statsimvastatin-study-results

20. Athauda D, Maclagan K, Skene SS, et al. Exenatide once weekly versus placebo in Parkinson's disease: a randomised, doubleblind, placebo-controlled trial. Lancet 2017;390:1664-1675.

21. Simuni T, Fiske B, Merchant K et al. Nilotinib in patients with advanced Parkinsons disease: a randomized phase 2A study (NILO-PD). https://www.medrxiv.org/content/10.1101/2020.05. 11.20093146v1. Accessed 22 Oct 2020. Preprint.

22. Pagan FL, Hebron ML, Wilmarth B, et al. Nilotinib Effects on Safety, Tolerability, and Potential Biomarkers in Parkinson Disease: A Phase 2 Randomized Clinical Trial. JAMA Neurol 2020;77:309-317.

23. Mullin S, Smith L, Lee K, et al. Ambroxol for the Treatment of Patients With Parkinson Disease With and Without Glucocerebrosidase Gene Mutations: A Nonrandomized, Noncontrolled Trial. JAMA Neurol 2020;77:427-434.

24. Polymeropoulos MH, Lavedan C, Leroy E, et al. Mutation in the alpha-synuclein gene identified in families with Parkinson's disease. Science 1997;276:2045-2047.

25. Spillantini MG, Schmidt ML, Lee VM, Trojanowski JQ, Jakes R, Goedert M. Alpha-synuclein in Lewy bodies. Nature 1997;388: 839-840.

26. Bendor JT, Logan TP, Edwards RH. The function of $\alpha$-synuclein. Neuron 2013;79:1044-1066.

27. Wong YC, Krainc D. $\alpha$-synuclein toxicity in neurodegeneration: mechanism and therapeutic strategies. Nat Med 2017;23:1-13.

28. Brás IC, Xylaki M, Outeiro TF. Mechanisms of alpha-synuclein toxicity: An update and outlook. Prog Brain Res 2020;252:91129.

29. Brundin P, Dave KD, Kordower JH. Therapeutic approaches to target alpha-synuclein pathology. Exp Neurol 2017;298:225-235.

30. Lewis J, Melrose H, Bumcrot D, et al. In vivo silencing of alphasynuclein using naked siRNA. Mol Neurodegener 2008;3:19.

31. Cooper JM, Wiklander PB, Nordin JZ, et al. Systemic exosomal siRNA delivery reduced alpha-synuclein aggregates in brains of transgenic mice. Mov Disord 2014;29:1476-1485.

32. Finkelstein DI, Billings JL, Adlard PA, et al. The novel compound PBT434 prevents iron mediated neurodegeneration and alphasynuclein toxicity in multiple models of Parkinson's disease. Acta Neuropathol Commun 2017;5:53.

33. Stamler D, Bradbury M, Wong C, Offman E. A first in human study of PBT434, a novel small molecule inhibitor of a-synuclein aggregation. Neurology 2019;92(suppl 15):S4.001. Abstract. 
34. Uemura N, Uemura MT, Luk KC, Lee VM, Trojanowski JQ. Cellto-Cell Transmission of Tau and $\alpha$-Synuclein. Trends Mol Med 2020;26:936-952.

35. Desplats P, Lee HJ, Bae EJ, et al. Inclusion formation and neuronal cell death through neuron-to-neuron transmission of alphasynuclein. Proc Natl Acad Sci U S A 2009;106:13010-13015.

36. Volpicelli-Daley LA, Luk KC, Patel TP, et al. Exogenous $\alpha$ synuclein fibrils induce Lewy body pathology leading to synaptic dysfunction and neuron death. Neuron 2011;72:57-71.

37. Luk KC, Kehm V, Carroll J, et al. Pathological $\alpha$-synuclein transmission initiates Parkinson-like neurodegeneration in nontransgenic mice. Science 2012;338:949-953.

38. Masuda-Suzukake M, Nonaka T, Hosokawa M, et al. Prion-like spreading of pathological $\alpha$-synuclein in brain. Brain 2013;136: $1128-1138$

39. Kordower JH, Chu Y, Hauser RA, Freeman TB, Olanow CW. Lewy body-like pathology in long-term embryonic nigral transplants in Parkinson's disease. Nat Med 2008;14:504-506.

40. Li JY, Englund E, Holton JL, et al. Lewy bodies in grafted neurons in subjects with Parkinson's disease suggest host-to-graft disease propagation. Nat Med 2008;14:501-503.

41. Chatterjee D, Kordower JH. Immunotherapy in Parkinson's disease: Current status and future directions. Neurobiol Dis 2019;132:104587.

42. Masliah E, Rockenstein E, Mante M, et al. Passive immunization reduces behavioral and neuropathological deficits in an alphasynuclein transgenic model of Lewy body disease. PLoS One 2011;6(4):e19338

43. Jankovic J, Goodman I, Safirstein B, et al. Safety and Tolerability of Multiple Ascending Doses of PRX002/RG7935, an Anti- $\alpha$ Synuclein Monoclonal Antibody, in Patients With Parkinson Disease: A Randomized Clinical Trial. JAMA Neurol 2018;75: 1206-1214.

44. Weihofen A, Liu Y, Arndt JW, et al. Development of an aggregate-selective, human-derived $\alpha$-synuclein antibody BIIB054 that ameliorates disease phenotypes in Parkinson's disease models. Neurobiol Dis 2019;124:276-288.

45. Brys M, Fanning L, Hung S, et al. Randomized phase I clinical trial of anti- $\alpha$-synuclein antibody BIIB054. Mov Disord 2019;34: $1154-1163$

46. Wouters OJ, McKee M, Luyten J. Estimated Research and Development Investment Needed to Bring a New Medicine to Market, 2009-2018. JAMA 2020;323:844-853.

47. Ashburn TT, Thor KB. Drug repositioning: identifying and developing new uses for existing drugs. Nat Rev Drug Discov 2004;3: 673-683.

48. Pushpakom S, Iorio F, Eyers PA, et al. Drug repurposing: progress, challenges and recommendations. Nat Rev Drug Discov 2019; 18:41-58.

49. Surmeier DJ, Obeso JA, Halladay GM. Selective neuronal vulnerability in Parkinson disease. Nat Rev Neurosci 2017;18:101-113.

50. Chan CS, Guzman JN, Ilijic E, et al. 'Rejuvenation' protects neurons in mouse models of Parkinson's disease. Nature 2007;447: 1081-1086.

51. Ilijic E, Guzman JN, Surmeier DJ. The L-type channel antagonist is neuroprotective in a mouse model of Parkinson's disease. Neurobiol Dis 2011;43:364-371.

52. Mullapudi A, Gudala K, Boya CS, Bansal D. Risk of Parkinson's disease in the users of antihypertensive agents: an evidence from the meta-analysis of observational studies. J Neurodegener Dis 2016;2016:5780809.

53. Parkinson Study Group. Phase II safety, tolerability, and dose selection study of isradipine as a potential disease-modifying intervention in early Parkinson's disease (STEADY-PD). Mov Disord 2013;28:1823-1831.
54. Jenner P. Oxidative stress in Parkinson's disease. Ann Neurol 2003;53 Suppl 3:S26-S38.

55. Schapira AH. Mitochondria in the aetiology and pathogenesis of Parkinson's disease. Lancet Neurol 2008;7:97-109.

56. Wang W, Wang X, Fujioka H, et al. Parkinson's diseaseassociated mutant VPS35 causes mitochondrial dysfunction by recycling DLP1 complexes. Nat Med 2016;22:54-63.

57. Parkinson Study Group. Effects of tocopherol and deprenyl on the progression of disability in early Parkinson's disease. N Engl J Med 1993;328:176-183.

58. Parkinson Study Group QE3 Investigators, Beal MF, Oakes D, et al. A randomized clinical trial of high-dosage coenzyme Q10 in early Parkinson disease: no evidence of benefit. JAMA Neurol 2014;71:543-552.

59. Pinto M, Nissanka N, Peralta S, Brambilla R, Diaz F, Moraes CT Pioglitazone ameliorates the phenotype of a novel Parkinson's disease mouse model by reducing neuroinflammation. Mol Neurodegener 2016;11:25.

60. NINDS Exploratory Trials in Parkinson Disease (NET-PD) FSZONE Investigators. Pioglitazone in early Parkinson's disease: a phase 2, multicentre, double-blind, randomised trial. Lancet Neurol 2015;14:795-803.

61. Crotty GF, Ascherio A, Schwarzschild MA. Targeting urate to reduce oxidative stress in Parkinson disease. Exp Neurol 2017;298:210-224.

62. Weisskopf MG, O'Reilly E, Chen H, Schwarzschild MA, Ascherio A. Plasma urate and risk of Parkinson's disease. Am J Epidemiol 2007;166:561-567.

63. Shen C, Guo Y, Luo W, Lin C, Ding M. Serum urate and the risk of Parkinson's disease: results from a meta-analysis. Can J Neurol Sci 2013;40:73-79.

64. Schwarzschild MA, Schwid SR, Marek K, et al. Serum urate as a predictor of clinical and radiographic progression in Parkinson disease. Arch Neurol 2008;65:716-723.

65. Ascherio A, LeWitt PA, Xu K, et al. Urate as a predictor of the rate of clinical decline in Parkinson disease. Arch Neurol 2009;66: 1460-1468.

66. Parkinson Study Group SURE-PD Investigators, Schwarzschild MA, Ascherio A, et al. Inosine to increase serum and cerebrospinal fluid urate in Parkinson disease: a randomized clinical trial. JAMA Neurol 2014;71:141-150.

67. Monti DA, Zabrecky G, Kremens D, et al. N-Acetyl Cysteine Is Associated With Dopaminergic Improvement in Parkinson's Disease. Clin Pharmacol Ther 2019;106:884-890.

68. Carroll CB, Wyse RKH. Simvastatin as a Potential DiseaseModifying Therapy for Patients with Parkinson's Disease: Rationale for Clinical Trial, and Current Progress. J Parkinsons Dis 2017;7:545-568

69. Selley ML. Simvastatin prevents 1-methyl-4-phenyl-1,2,3,6tetrahydropyridine-induced striatal dopamine depletion and protein tyrosine nitration in mice. Brain Res 2005;1037:1-6.

70. Ghosh A, Roy A, Matras J, Brahmachari S, Gendelman HE, Pahan K. Simvastatin inhibits the activation of $\mathrm{p} 21$ ras and prevents the loss of dopaminergic neurons in a mouse model of Parkinson's disease. J Neurosci 2009;29:13543-13556.

71. Moreau C, Duce JA, Rascol O, et al. Iron as a therapeutic target for Parkinson's disease. Mov Disord 2018;33:568-574.

72. Guiney SJ, Adlard PA, Bush AI, Finkelstein DI, Ayton S. Ferroptosis and cell death mechanisms in Parkinson's disease. Neurochem Int 2017;104:34-48.

73. Devos D, Moreau C, Devedjian JC, et al. Targeting chelatable iron as a therapeutic modality in Parkinson's disease. Antioxid Redox Signal 2014;21:195-210.

74. Martin-Bastida A, Ward RJ, Newbould R, et al. Brain iron chelation by deferiprone in a phase 2 randomised double-blinded 
placebo controlled clinical trial in Parkinson's disease. Sci Rep 2017;7:1398.

75. Athauda D, Foltynie T. The glucagon-like peptide 1 (GLP) receptor as a therapeutic target in Parkinson's disease: mechanisms of action. Drug Discov Today 2016;21:802-818.

76. Athauda D, Foltynie T. Insulin resistance and Parkinson's disease: A new target for disease modification? Prog Neurobiol 2016;145146:98-120.

77. De Pablo-Fernandez E, Goldacre R, Pakpoor J, Noyce AJ, Warner TT. Association between diabetes and subsequent Parkinson disease: A record-linkage cohort study. Neurology 2018;91:e139e142.

78. Cereda E, Barichella M, Pedrolli C, et al. Diabetes and risk of Parkinson's disease: a systematic review and meta-analysis. Diabetes Care 2011;34:2614-2623.

79. Hu G, Jousilahti P, Bidel S, Antikainen R, Tuomilehto J. Type 2 diabetes and the risk of Parkinson's disease. Diabetes Care 2007;30:842-847.

80. Lu L, Fu DL, Li HQ, Liu AJ, Li JH, Zheng GQ. Diabetes and risk of Parkinson's disease: an updated meta-analysis of case-control studies. PLoS One 2014;9:e85781.

81. Palacios N, Gao X, McCullough ML, et al. Obesity, diabetes, and risk of Parkinson's disease. Mov Disord 2011;26:2253-2259.

82. Xu Q, Park Y, Huang X, et al. Diabetes and risk of Parkinson's disease. Diabetes Care 2011;34:910-915.

83. Parkes DG, Mace KF, Trautmann ME. Discovery and development of exenatide: the first antidiabetic agent to leverage the multiple benefits of the incretin hormone, GLP-1. Expert Opin Drug Discovery 2013;8:219-244.

84. Yun SP, Kam TI, Panicker N, et al. Block of A1 astrocyte conversion by microglia is neuroprotective in models of Parkinson's disease. Nat Med 2018;24:931-938.

85. Aviles-Olmos I, Dickson J, Kefalopoulou Z, et al. Exenatide and the treatment of patients with Parkinson's disease. J Clin Invest 2013;123:2730-2736.

86. Brahmachari S, Karuppagounder SS, Ge P, et al. c-Abl and Parkinson's Disease: Mechanisms and Therapeutic Potential. J Parkinsons Dis 2017;7:589-601

87. Brahmachari S, Ge P, Lee SH, et al. Activation of tyrosine kinase c-Abl contributes to $\alpha$-synuclein-induced neurodegeneration. J Clin Invest 2016;126:2970-2988.

88. Karuppagounder SS, Brahmachari S, Lee Y, Dawson VL, Dawson TM, Ko HS. The c-Abl inhibitor, nilotinib, protects dopaminergic neurons in a preclinical animal model of Parkinson's disease. Sci Rep 2014;4:4874.

89. Pagan F, Hebron M, Valadez EH, et al. Nilotinib Effects in Parkinson's disease and Dementia with Lewy bodies. J Parkinsons Dis 2016;6:503-517.

90. Schwarzschild MA. Could MAO-B inhibitor withdrawal rather than nilotinib benefit explain the dopamine metabolite increase in Parkinsonian study subjects? J Parkinsons Dis 2017;7:79-80.

91. ClinicalTrials.gov [Internet]. Bethesda (MD): National Library of Medicine (US). 2009 Feb 2 -. Identifier NCT00833690. Study of Urate Elevation in Parkinson's Disease (SURE-PD); 2014 Jun 5 [cited 2020 Oct 22]. Available from: https://clinicaltrials.gov/ct2/ show/NCT00833690

92. Lang AE, Melamed E, Poewe W, Rascol O. Trial designs used to study neuroprotective therapy in Parkinson's disease. Mov Disord 2013;28:86-95.
93. Thibault L, Rascol O, Corvol JC, et al. New perspectives on study designs for evaluating neuroprotection in Parkinson's disease. Mov Disord 2017;32:1365-1370.

94. Olanow CW, Kieburtz K, Katz R. Clinical approaches to the development of a neuroprotective therapy for PD. Exp Neurol 2017;298(Pt B):246-251.

95. Fayyad M, Salim S, Majbour N, et al. Parkinson's disease biomarkers based on $\alpha$-synuclein. J Neurochem 2019;150:626-636.

96. Brooks DJ, Tambasco N. Imaging synucleinopathies. Mov Disord 2016;31:814-29.

97. The Michael J. Fox Foundation. Michael J. Fox Foundation Announces Winners of the "Ken Griffin Alpha-synuclein Imaging Competition" to Develop Game-Changing Tool for Parkinson's Research [Internet]. New York: The Michael J. Fox Foundation; 30 Jul 2020 [cited 2020 Oct 22]. Available from: https://www.michaeljfox.org/publication/michael-j-foxfoundation-announces-winners-ken-griffin-alpha-synucleinimaging

98. Stephenson D, Hill D, Cedarbaum JM, et al. The Qualification of an Enrichment Biomarker for Clinical Trials Targeting Early Stages of Parkinson's Disease. J Parkinsons Dis 2019;9:553-563.

99. Marek K, Seibyl J, Eberly S, et al. Longitudinal follow-up of SWEDD subjects in the PRECEPT Study. Neurology 2014;82: 1791-1797.

100. Erro R, Schneider SA, Stamelou M, Quinn NP, Bhatia KP. What do patients with scans without evidence of dopaminergic deficit (SWEDD) have? New evidence and continuing controversies. J Neurol Neurosurg Psychiatry 2016;87:319-323.

101. Conrado DJ, Nicholas T, Tsai K, et al. Dopamine Transporter Neuroimaging as an Enrichment Biomarker in Early Parkinson's Disease Clinical Trials: A Disease Progression Modeling Analysis. Clin Transl Sci 2018;11:63-70.

102. Suwijn SR, Samim H, Eggers C, et al. Value of Clinical Signs in Identifying Patients with Scans without Evidence of Dopaminergic Deficit (SWEDD) [published online ahead of print, 2020 Jun 25]. J Parkinsons Dis. 2020. https://doi.org/10.3233/ JPD-202090.

103. Mahlknecht P, Seppi K, Poewe W. The Concept of Prodromal Parkinson's Disease. J Parkinsons Dis 2015;5:681-697.

104. Postuma RB, Berg D. Advances in markers of prodromal Parkinson disease. Nat Rev Neurol 2016;12:622-634.

105. Postuma RB, Berg D. Prodromal Parkinson's Disease: The Decade Past, the Decade to Come. Mov Disord 2019;34:665-675.

106. Goetz CG, Tilley BC, Shaftman SR, et al. Movement Disorder Society-sponsored revision of the Unified Parkinson's Disease Rating Scale (MDS-UPDRS): scale presentation and clinimetric testing results. Mov Disord 2008;23:2129-2170.

107. Biglan KM, Oakes D, Lang AE, et al. A novel design of a Phase III trial of isradipine in early Parkinson disease (STEADY-PD III). Ann Clin Transl Neurol 2017;4:360-368.

108. Espay AJ, Bonato P, Nahab FB, et al. Technology in Parkinson's disease: Challenges and opportunities. Mov Disord 2016;31: 1272-1282.

109. Espay AJ, Hausdorff JM, Sánchez-Ferro Á, et al. A roadmap for implementation of patient-centered digital outcome measures in Parkinson's disease obtained using mobile health technologies. Mov Disord 2019;34:657-663.

Publisher's Note Springer Nature remains neutral with regard to jurisdictional claims in published maps and institutional affiliations. 